\title{
TIME-PERIODIC WAVE LOADING ON A SUBMERGED \\ CIRCULAR CYLINGER IN A CURRENT
}

\author{
by \\ John Grue \\ Department of Mechanics, \\ University of Oslo, \\ Blindern, 0316 oslo 3, Norway
}

\section{Abstract.}

The time-periodic pressure loading, the added mass, damping and exitation forces on a horizontal submerged circular cylinder in a current are examined. The fluid layer is infinitely deep and the motion is two-dimensional. The boundary value problem is solved by applying a source distribution along the contour of the body. The forces become finite for $\tau=U \sigma / g$ approaching $1 / 4$. (U speed of the current, $\sigma$ frequency, $g$ acceleration due to gravity.) The added mass force becomes negative for $\tau$ close to 1/4. The damping force is very small for $\tau>1 / 4$. The exitation loading on the cylinder is larger for incoming waves travelling against the current than for incoming waves travelling with the current. 



\section{Introduction.}

We shall consider wave loads on an infinitely long horizontal circular cylinder, submerged under a free surface in a uniform current. The cylinder can undergo oscillations in sway or heave, or be embedded in incoming harmonic waves. We shall be interested in the time periodic wave loads. The current is horizontal and normal to the generators of the cylinder. The problem may then be considered as two-dimensional. The fluid layer is of infinite depth and of infinite lateral extent. The fluid is assumed incompressible and the motion irrotational. All equations are linearized.

Many offshore constructions are built up of long cylinders. Examples are oil platforms, with brazings and cylindrical pontoons, and pipelines. Computation of wave loads on long cylinders is therefore important in investigating the behaviour of many offshore constructions in waves. In most practical situations of wave scattering from a body on the ocean a current may also be present. The theory and computations presented here are appropriate in predicting fluid loading on horizontal submerged cylinders when the crests of the incoming waves are parallel to the cylinder axis.

Two-dimensional wave scattering from a submerged circular cylinder in the absence of a current has been treated by Dean (1948), Ursell (1950), Ogilvie (1963), Grue and Palm (1984) and Vada (1985). In Grue and Palm (1985) the radiation problem and the diffraction problem have been generalized to allow for a uniform current. For the benefit of new readers we shortly describe the properties of the wave-field and the results obtained in Grue and Palm (1985), which is the basis of the present paper. 
The uniform current gives rise to stationary lee waves behind the cylinder. These waves are treated by e.g. Lamb (1932), Havelock (1936) and Tuck (1965). We shall here only be interested in the lee wave field to account for interactions with the time periodic wave field. The current may also, as well as the oscillatory wave motion, generate vortex shedding. These effects are disregarded in the calculations.

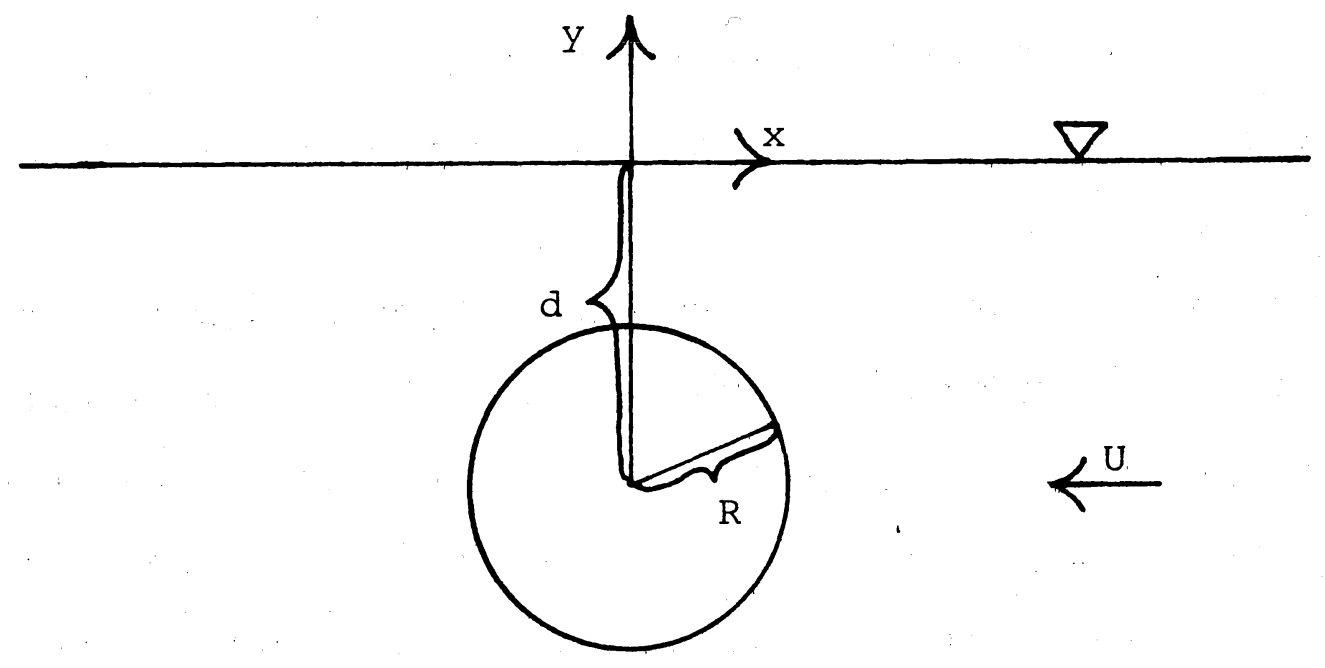

Figure 1. Definition sketch

We introduce cartesian axes $0 x y$ with origin 0 in the undisturbed free surface, $0 x$ directed horizontal and normal to the generators of the cylinder, and $0 y$ positive upwards. The velocity $U$ of the uniform current is along the negative $x$-axis. If the cylinder is oscillating with a frequency $\sigma \quad(\sigma>0)$ in the current, four travelling waves are present when $\tau=U \sigma / g<l / 4 \quad(g$ the acceleration due to gravity) and only two waves when $\tau>1 / 4$. The wave numbers of the four waves are given by

$$
\sigma=\sqrt{\mathrm{gk}}_{1,2}-\mathrm{Uk}_{1,2} \quad \tau<1 / 4
$$


1. Introduction.

We shall consider wave loads on an infinitely long horizontal circular cylinder, submerged under a free surface in a uniform current. The cylinder can undergo oscillations in sway or heave, or be embedded in incoming harmonic waves. We shall be interested in the time periodic wave loads. The current is horizontal and normal to the generators of the cylinder. The problem may then be considered as two-dimensional. The fluid layer is of infinite depth and of infinite lateral extent. The fluid is assumed incompressible and the motion irrotational. All equations are linearized.

Many offshore constructions are built up of long cylinders. Examples are oil platforms, with brazings and cylindrical pontoons, and pipelines. Computation of wave loads on long cylinders is therefore important in investigating the behaviour of many offshore constructions in waves. In most practical situations of wave scattering from a body on the ocean a current may also be present. The theory and computations presented here are appropriate in predicting fluid loading on horizontal submerged cylinders when the crests of the incoming waves are parallel to the cylinder axis.

Two-dimensional wave scattering from a submerged circular cylinder in the absence of a current has been treated by Dean (1948), Ursell (1950), Ogilvie (1963), Grue and Palm (1984) and Vada (1985). In Grue and Palm (1985) the radiation problem and the diffraction problem have been generalized to allow for a uniform current. For the benefit of new readers we shortly describe the properties of the wave-field and the results obtained in Grue and Palm (1985), which is the basis of the present paper. 
The uniform current gives rise to stationary lee waves behind the cylinder. These waves are treated by e.g. Lamb (1932), Havelock (1936) and Tuck (1965). We shall here only be interested in the lee wave field to account for interactions with the time periodic wave field. The current may also, as well as the oscillatory wave motion, generate vortex shedding. These effects are disregarded in the calculations.

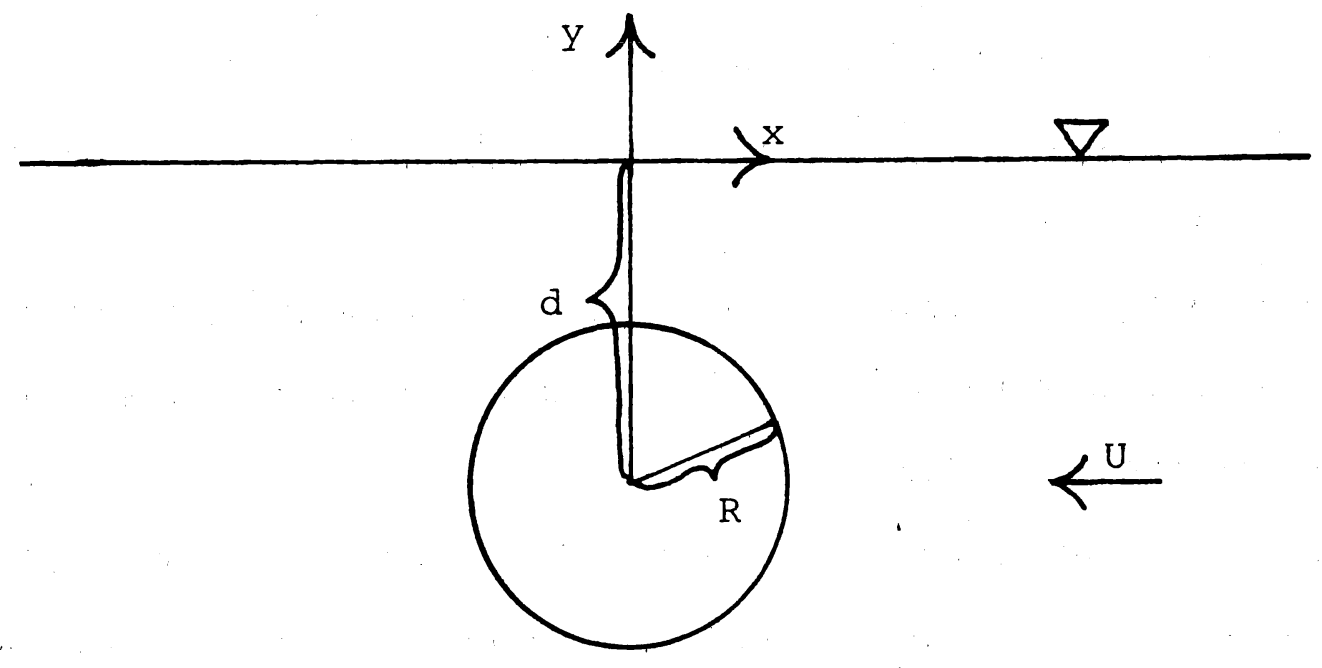

Figure 1. Definition sketch

We introduce cartesian axes $0 x y$ with origin 0 in the undisturbed free surface, $0 x$ directed horizontal and normal to the generators of the cylinder, and $0 y$ positive upwards. The velocity $U$ of the uniform current is along the negative $x$-axis. If the cylinder is oscillating with a frequency $\sigma \quad(\sigma>0)$ in the current, four travelling waves are present when $\tau=U \sigma / g<1 / 4$ (g the acceleration due to gravity) and only two waves when $\tau>1 / 4$. The wave numbers of the four waves are given by

$$
\sigma={\sqrt{g k_{1}}}_{1,2}-\mathrm{Uk}_{1,2} \quad \tau<1 / 4
$$




$$
\sigma=\mathrm{Uk}_{3,4} \mp \sqrt{\mathrm{gk}} 3,4
$$

The $\mathrm{k}_{2}$-wave is travelling uptream with positive phase and group velocity. The $\mathrm{k}_{1}$-wave, however, is travelling downstream with positive phase velocity but with negative group velocity. The $\mathrm{k}_{3}$-wave and $\mathrm{k}_{4}$-wave are travelling downstream with negative phase and group velocities. In the frame of reference where the speed of the current is zero, the relative frame of reference, the $k_{1}-$, $\mathrm{k}_{2}$ - and $\mathrm{k}_{3}$-waves are travelling towards $\mathrm{x}=\infty$, whereas the $\mathrm{k}_{4}-$ wave is travelling towards $x=-\infty$.

The solution of the boundary value problem was obtained by expressing the velocity potential as a distribution of wave sources along the contour. The unknown source strength was determined by an integral equation, which yielded a unique solution. The amplitudes of the scattered waves were then easily computed. In the radiation problem it was shown that the amplitudes of the $k_{1}$-wave and $k_{2}$-wave approached a finite limit as $\tau \rightarrow 1 / 4$, which means that the energy flux of the $k_{1}$ - and $k_{2}$-waves tends to zero as $\tau \rightarrow 1 / 4$. The damping force, which is related to the energy flux of the outgoing waves, then tends to a finite value as $\tau \rightarrow 1 / 4$. In the diffraction problem it was shown that one incoming wave gives rise to one new wave only (and not three new waves, which is the case for non-circular contours). The mean horizontal second order forces in the radiation and diffraction problems were obtained by the momentum equation.

In the present paper we apply the mathematical method sketched above to account for the pressure loading at the cylinder, the added mass, damping and exitation forces on the cylinder. In the radiation problem the loading becomes finite as 
$\tau \rightarrow 1 / 4$. The added mass force becomes negative for $\tau$ close to 1/4. It is well known that for $U=0$ there are no forces orthogonal to the swaying or heaving motion of the cylinder. This is, however, not true when a current is present. For moderate values of $U$ it is found that the vertical force in sway and the horizontal force in heave (with same amplitude of oscillation) are equal in magnitude and of opposite sign. In the diffraction problem the loading at the cylinder approaches zero when the group velocity of the incoming wave tends to zero $(\tau \rightarrow] / 4)$. The exitation loading is larger for incoming wave travelling against the current $\left(\mathrm{k}_{2}\right.$-wave) than for incoming wave travelling with the current $\left(\mathrm{k}_{4}\right.$-wave).

2. Mathematical formulation.

According to the previous assumptions, the velocity of the flutd is given by

$$
\vec{v}=-U\left(\vec{e}_{1}+\nabla \chi(x, y)\right)+\nabla \Phi(x, y, t)
$$

where $\vec{e}_{l}$ is the unit vector along $0 x, \chi$ is the stationary lee wave potential, $\Phi$ is the time dependent potential and $t$ denotes time. Assume that the cylinder can óscillate with its centre at

$$
\vec{e}_{1}+(y+d) \vec{e}_{2}=\operatorname{Re}(\vec{\xi} \exp (j \sigma t))
$$

where $d$ is the depth of the cylinder centre, $\vec{e}_{2}$ is the unit vector along $0 y, R e$ denotes the real pant, $j$ is the imaginary unit, and $\vec{\xi}$ is given by 


$$
\vec{\xi}=\xi_{1} \vec{e}_{1}+\xi_{2} \vec{e}_{2}
$$

We assume that $\vec{\xi}$ and the amplitude of the incoming wave are small so that the boundary value problem for $\Phi$ may be linearized. We also assume that the boundary value problem for $\chi$ may be linearized. This assumption is assymptotic valid for large $d / R$. ( $R$ the radius of the circle.) Writing

$$
\Phi(x, y, t)=\operatorname{Re} \phi(x, y) \exp (j \sigma t) \quad(\sigma>0)
$$

the velocity potentials $\chi$ and $\phi$ fulfill the following linear boundary value problems

$$
\nabla^{2} \chi=0 \quad \text { in the fluid domain }
$$

subject to

$$
\begin{array}{ll}
\mathrm{U}^{2} \frac{\partial^{2} \chi}{\partial \mathrm{x}^{2}}+9 \frac{\partial \chi}{\partial y}=0 & y=0 \\
\frac{\partial \chi}{\partial \mathrm{n}}=-\mathrm{n}_{1} & \text { at } \mathrm{S} \\
\chi \rightarrow 0 & \mathrm{x} \rightarrow+\infty \\
\nabla \chi \rightarrow 0 & \mathrm{y} \rightarrow-\infty
\end{array}
$$

$$
\nabla^{2} \phi=0 \quad \text { in the fluid domain }
$$

subject to

$$
\begin{aligned}
& \left(j \sigma-U \frac{\partial}{\partial x}\right)^{2} \phi+g \frac{\partial \phi}{\partial y}=0 \quad y=0 \\
& \frac{\partial \phi}{\partial n}=\vec{n} \cdot(j \sigma \vec{\xi}+U(\vec{\xi} \cdot \nabla) \nabla \chi) \text { at } S \\
& \text { radiation conditions at } x= \pm \infty \\
& \nabla \phi \rightarrow 0 \quad y \rightarrow-\infty
\end{aligned}
$$


Here $\partial / \partial \mathrm{n}$ denotes the normal derivative, and $\mathrm{n}_{1}$ is the $\mathrm{x}-$ component of the normal vector $\vec{n}$ of the body, directed into the fluid. The mean position of the cylinder contour is denoted by S. The condition $(2.6 \mathrm{c})$ is obtained by Taylor expansion of the total velocity potential about $S$. The proper radiation conditions in (2.6d) are obtained from Haskind (1954) as one outgoing $\mathrm{k}_{2}$-wave with phase velocity $\sigma / \mathrm{k}_{2}$ at $\mathrm{x}=+\infty$ and one incoming $k_{1}$-wave, one outgoing $k_{3}$-wave, one outgoing $k_{4}$-wave, with phase velocities $\sigma / \mathrm{k}_{1}, \sigma / \mathrm{k}_{3}, \sigma / \mathrm{k}_{4}$, respectively, at $\mathbf{x}=-\infty$.

Let now

$$
\phi=\phi_{0}+\phi_{1}
$$

where $\phi_{0}$ is considered as known and $\phi_{l}$ is unknown. In the radiation problem $\phi_{0} \equiv 0$. In the diffraction problem $\phi_{0}$ is the potential of the incoming wave. The potentials $\chi$ and $\phi_{1}$ are both given by a source distribution along the contour $S$. The local source strength for $\chi$ or $\phi_{1}$ is then obtained from integral equations, as described in Grue and Palm (1985).

3. The pressure and the pressure forces.

Let the mean position of the contour, S, be parameterized by

$$
\begin{aligned}
& x=-R \sin \theta \\
& y=-d+R \cos \theta
\end{aligned}
$$

The uppermost point of the cylinder corresponds to $\theta=0$. The pressure at a point within the fluid is given by the Bernoulli equation ( $p=0$ at the free surface).

$$
p=-\rho\left(\frac{\partial \Phi}{\partial t}+\frac{1}{2}\left(|\vec{v}|^{2}-U^{2}\right)+g y\right)
$$


$\rho$ is the density of the fluid. The pressure at a point at the instantaneous position $\hat{S}$ of the cylinder contour is obtained by Taylor expansion about $\mathrm{S}$. By retaining terms which are linear in $\Phi$ and $\vec{\xi}$ we obtain

$$
p=\operatorname{Re} p_{1} \exp (j \sigma t)+p_{0}
$$

where

$$
p_{1}=-\rho\left(j \sigma \phi+v_{\theta} \frac{1}{R} \frac{\partial \phi}{\partial \theta}+\frac{1}{2} \vec{\xi} \cdot \nabla v_{\theta}^{2}+\xi_{2} g\right)
$$

and

$$
\begin{aligned}
& \mathrm{p}_{0}=-\rho\left(\frac{1}{2}\left(\mathrm{v}_{\theta}^{2}-\mathrm{u}^{2}\right)+\mathrm{gy}\right) \\
& \mathrm{v}_{\theta}=-\frac{\mathrm{U}}{\mathrm{R}} \frac{\partial}{\partial \theta}(\mathrm{x}+\chi) \quad \text { (at the contour) }
\end{aligned}
$$

The pressure force on the body is given by

$$
\vec{F}=\int_{\hat{S}}-p \vec{p} d s
$$

By Taylor expansion the part of the force oscillating with frequency $\sigma$ is obtained as

$$
\vec{F}=\operatorname{Re} \vec{P} \exp (j \sigma t)
$$

where

$$
\vec{P}=\int_{S}-p_{1} \vec{n} d S
$$

We note that the last two terms in (3.3a) contributes to the pressure and the pressure force only in the radiation problem. 
3a. The radiation problem.

We shall first consider the radiation problem. There are then no incident waves, i.e. $\phi_{0} \equiv 0$. The components of $\vec{\xi}$ are given by $\xi_{1}=\varepsilon, \xi_{2}=0$ in sway, and $\xi_{1}=0, \xi_{2}=\varepsilon$ in heave, $\varepsilon$ is real. In sway the force may be written by

$$
\vec{P}=\left(\hat{\mu}_{11}-j \hat{\lambda}_{11}\right) \vec{e}_{1}+\left(\hat{\mu}_{21}-j \hat{\lambda}_{21}\right) \vec{e}_{2}
$$

and in heave by

$$
\vec{P}=\left(\hat{\mu}_{12}-j \hat{\lambda}_{12}\right) \vec{e}_{1}+\left(\hat{\mu}_{22}-j \hat{\lambda}_{22}\right) \vec{e}_{2}
$$

where $\hat{\mu}_{m n}$ and $\hat{\lambda}_{m n}$ are real, being the components of the added mass force and the damping force, respectively. Non-dimensional forces are obtained as follows

$$
\mu_{m n}=\hat{\mu}_{m n} / \rho g R \varepsilon, \quad \lambda_{m n}=\hat{\lambda}_{m n} / \rho g R \varepsilon
$$

Values of $\mu_{m n}$ and $\lambda_{m n}$ are displayed in figure 2 for $U=0$ and in figures $3(a, c)$ for $U / \sqrt{g R}=0.4$. In all cases $d / R=2$. The computations show that the three first terms in (3.3a) give significant contributions to $\mu_{\mathrm{mn}}$ and $\lambda_{\mathrm{mn}}$. For $\mathrm{U}=0$ we have

$$
\begin{array}{ll}
\mu_{11}=\mu_{22} & \lambda_{11}=\lambda_{22} \\
\mu_{m n}=0, & \lambda_{m n}=0, \quad m \neq n
\end{array}
$$

The occurrence of a current changes the values of $\mu_{\mathrm{mn}}$ and $\lambda_{\mathrm{mn}}$ considerably. Now there are forces which are orthogonal to the swaying or heaving motion of the cylinder, and $\mu_{11}-j \lambda_{11}$ is

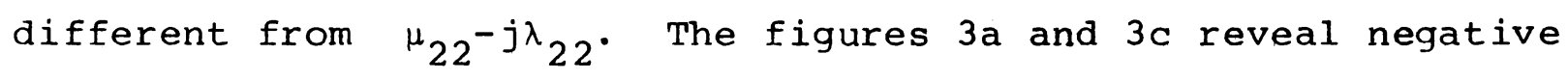
values of the added mass forces $\mu_{\mathrm{mm}}$ when $\tau$ is close to $1 / 4$. We note that the damping forces $\lambda_{\mathrm{mm}}$ are small for $\tau>1 / 4$. The 


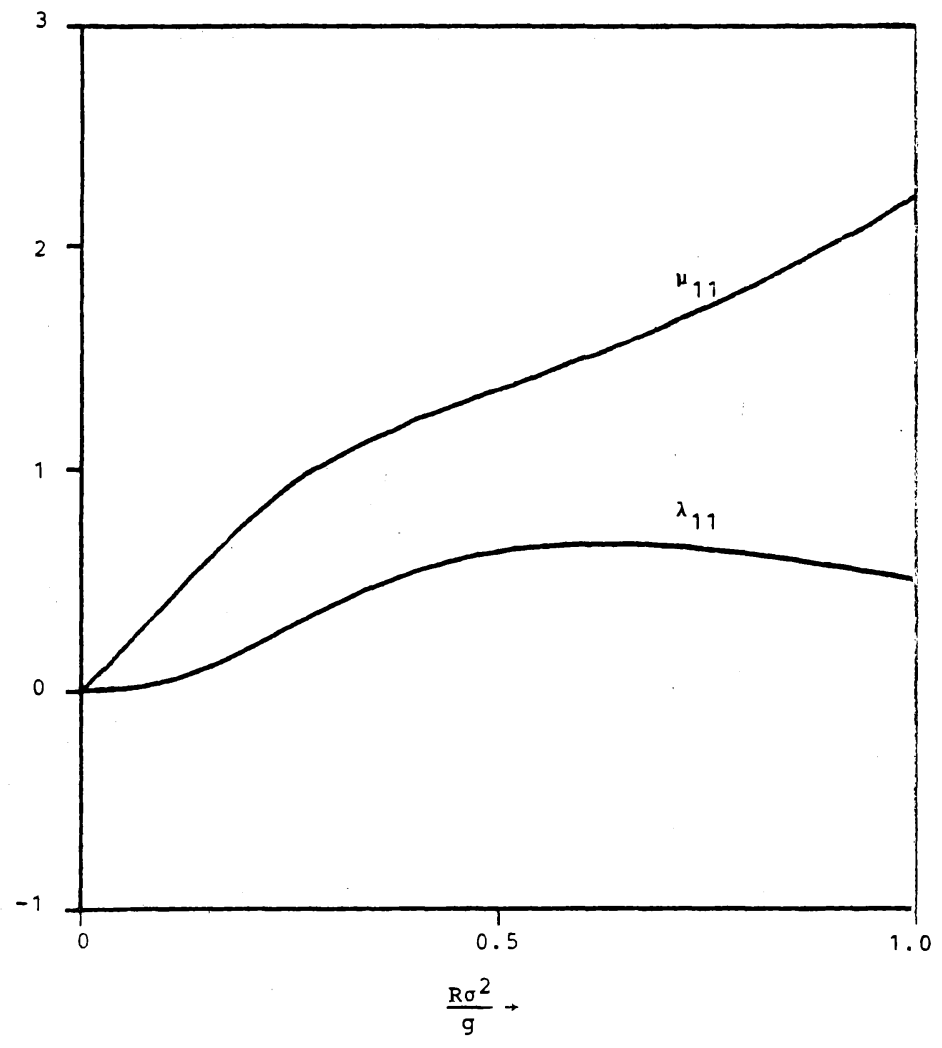

Figure 2. Added mass force $\mu_{11}$ and damping force $\lambda_{11}, U=0, d / R=2$.

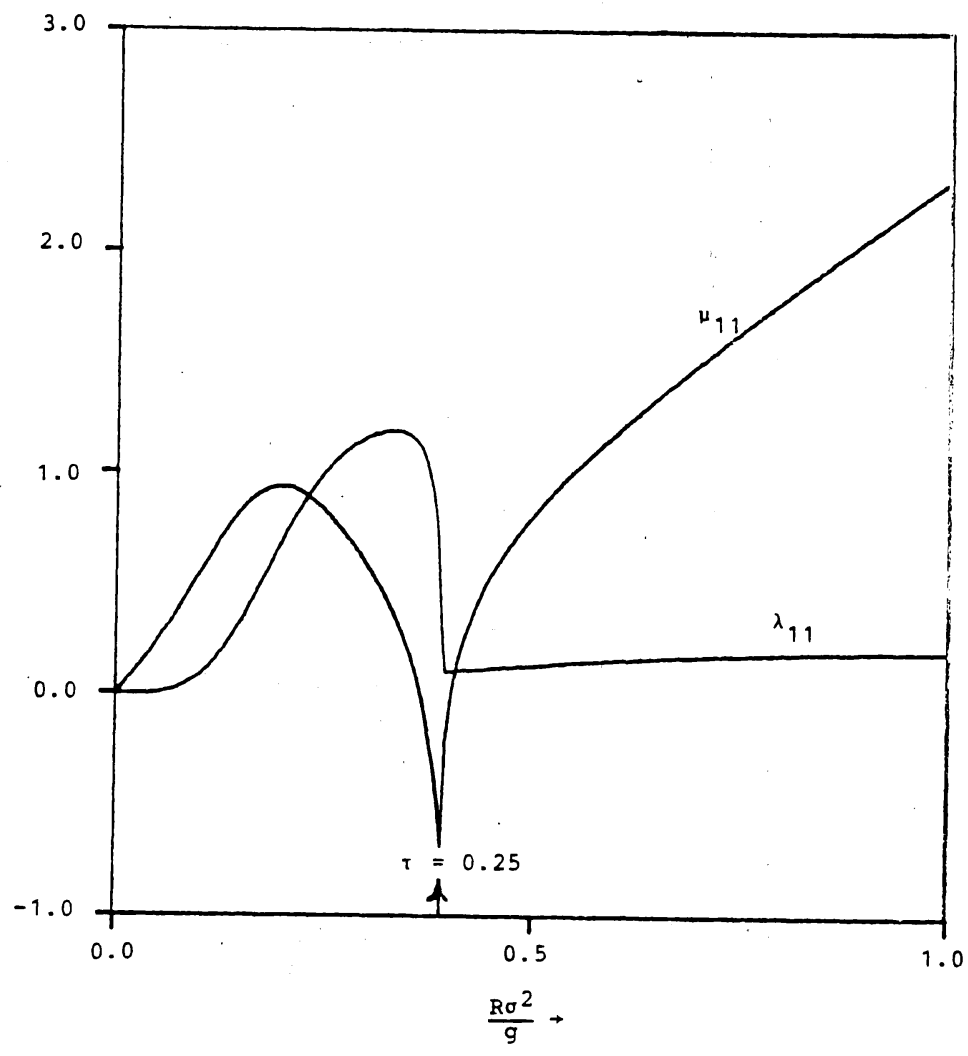

(3a) 


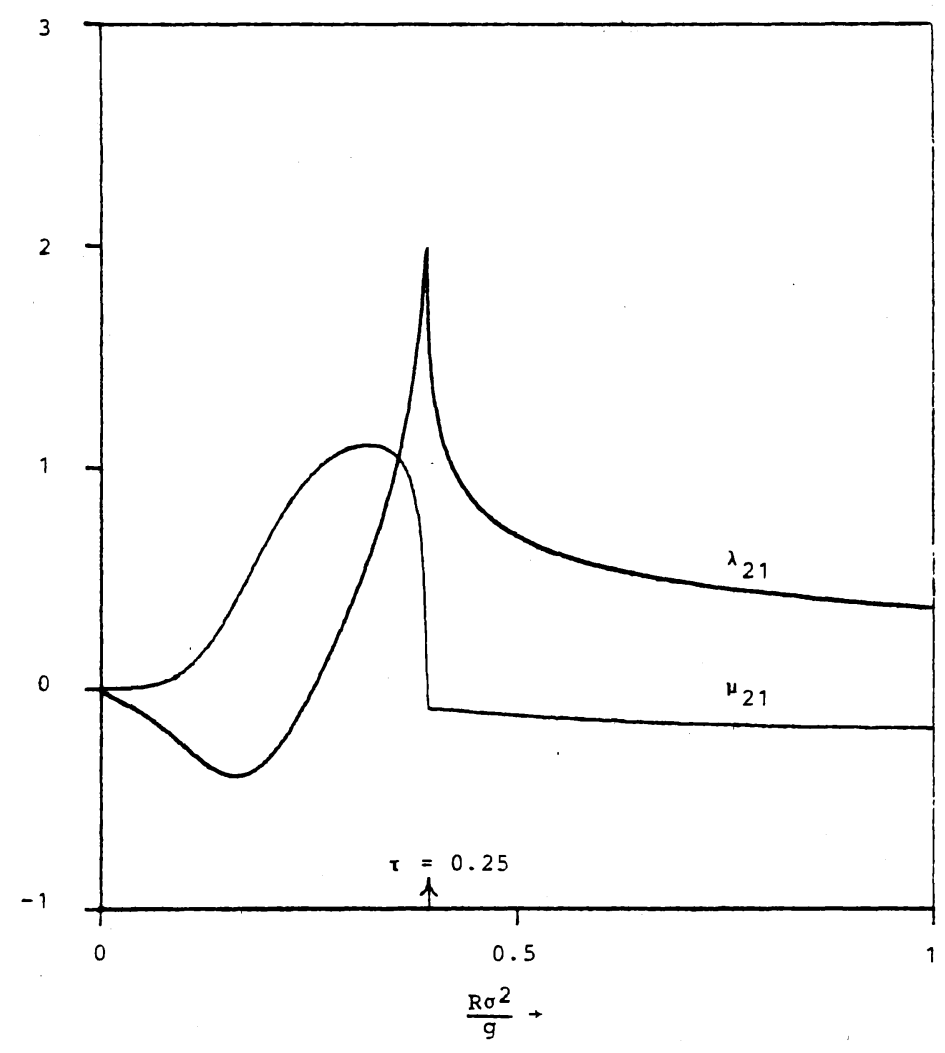

(3b)

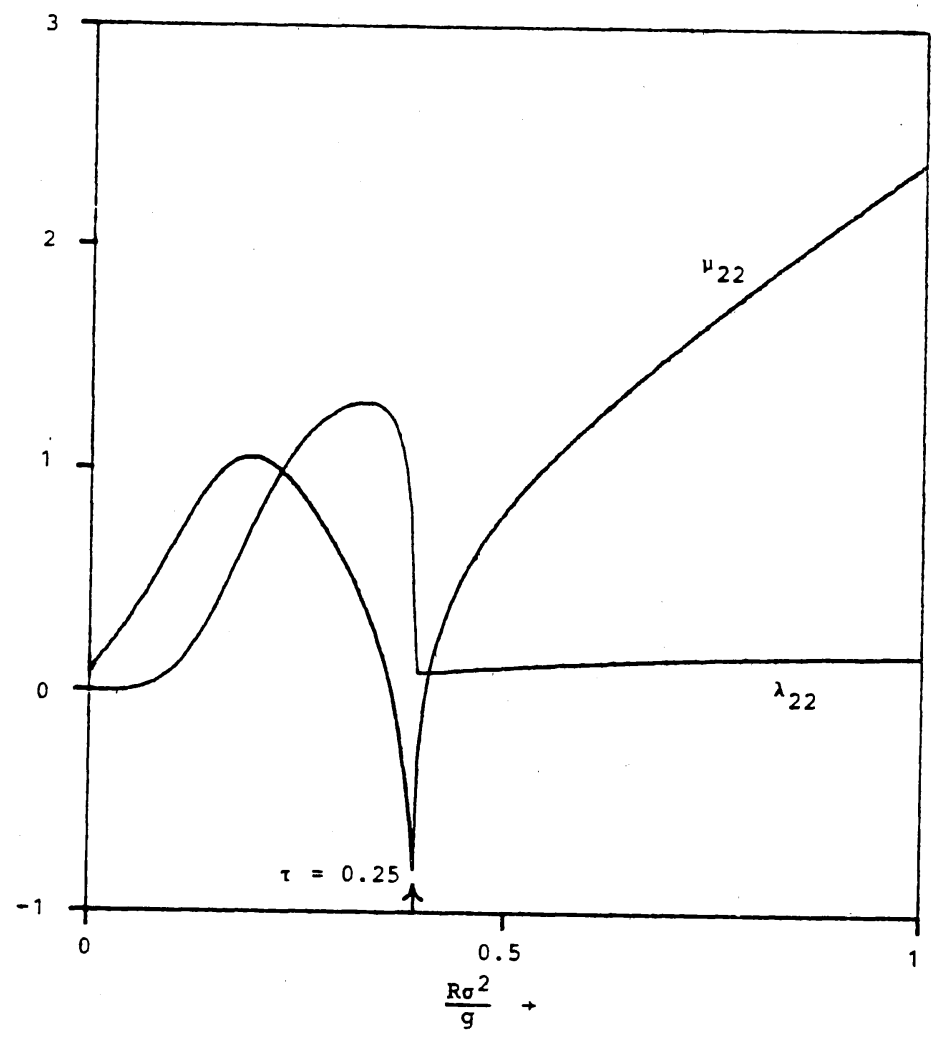

(3c)

Figure 3. Added mass forces and damping forces, $U / \sqrt{g R}=0.4, d / R=2$. 
added mass and damping forces remain finite for $\tau$ approaching 1/4. In Mo and Palm (1985) this result is also obtained for a cylinder of elliptic shape. This is in disagreement with e.g. Sclavounos (1984) where added mass and damping forces are obtained for a floating 3-D ship. However, the force components vary rather rapidly for $\tau$ close to $1 / 4$. Some of the components have also a peak for $\tau$ approaching $1 / 4$. For the speeds of the current considered here, i.e. $\mathrm{U} / \sqrt{\mathrm{gR}}<0.6$, the amplitude of the lee waves is approximately zero and the lee wave potential $\chi$ becomes approximately anti-symmetric with respect to $x=0$, i.e.

$$
\chi(x, y)=-\chi(-x, y)
$$

It is numerically confirmed that the cross coupling forces are anti-symmetric when $(3.11)$ is fulfilled, i.e.

$$
\mu_{21}=-\mu_{12}, \quad \lambda_{21}=-\lambda_{12}
$$

For $U / \sqrt{g R}=0.4$ and $d / R=2,(3.12)$ is fulfilled within an accuracy of less than 1 pr. cent.

In figure 4 the amplitude of the pressure distribution along the cylinder contour is displayed for heaving motion. The maximum pessure is slightly larger for $U / \sqrt{g R}=0.4$ than for $U=0$. The pressure distribution is symmetric about $\theta=0$ for $U=0$. This is, however, not true for $U / \sqrt{\mathrm{gR}}=0.4$. 


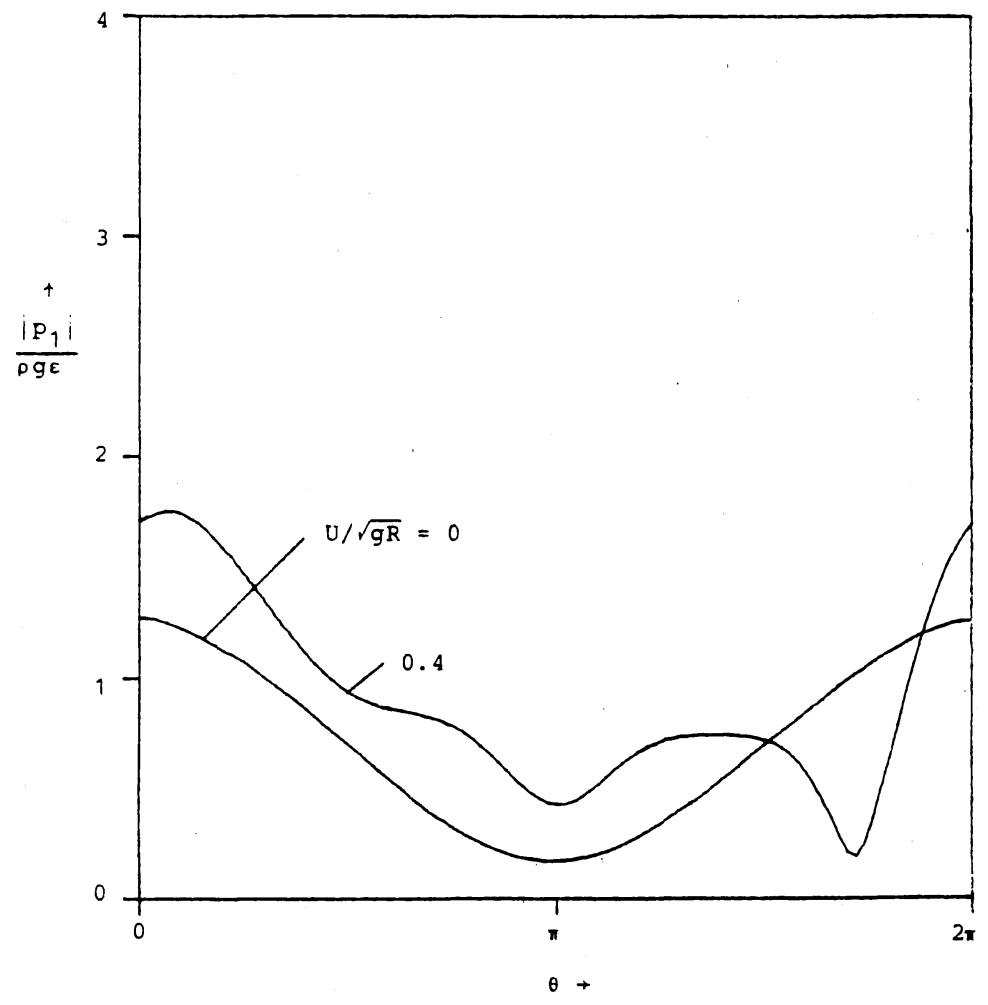

Figure 4. Pressure distribution along the circular contour when the cylinder is oscillating in heave, $\mathrm{d} / \mathrm{R}=2, \mathrm{R} \sigma^{2} / \mathrm{g}=0.65$ and $\mathrm{U}=0, \mathrm{R \sigma ^{2 }} / \mathrm{g}=0.33$ and $\mathrm{U} / \sqrt{\mathrm{gR}}=0.4$. 
3b. The diffraction problem.

Let us then consider the diffraction problem. Now $\vec{\xi}=0$, and $\phi_{0}$ is given by

$$
\phi_{0}(x, y)=\delta a \sqrt{\frac{g}{k}} \exp (k y \mp j k x)
$$

where $\delta=-1$ for incoming $k_{1}, k_{2}, k_{3}$ waves, i.e. waves travelling along the positive $\mathrm{x}$-direction in the relative frame of reference, hereafter denoted by waves travelling against the current, and $\delta=1$ for $\mathrm{k}_{4}$-waves, i.e. waves travelling along the negative $\mathrm{x}$-direction in the relative frame of reference, hereafter denoted by waves travelling with the current. The amplitude of the incident waves is $a, k$ is the wave number, the - sign corresponds to incoming $\mathrm{k}_{1}, \mathrm{k}_{2}$-waves and the + sign corresponds to incoming $\mathrm{k}_{3}, \mathrm{k}_{4}$-waves. The components of the exitation force are given by

$$
\vec{P}=\hat{x}_{1} \vec{e}_{1}+\hat{x}_{2} \vec{e}_{2}
$$

The exitation forces are non-dimensionalized by

$$
\mathrm{x}_{\mathrm{m}}=\hat{\mathrm{x}}_{\mathrm{m}} / \rho g R a
$$

The exitation forces for incoming waves travelling with the current are displayed in figures $5(a, b)$. The exitation force is of the same order of magnitude for $U=0$ and $U / \sqrt{g R}=0.4$. We note that $\left|x_{1}\right|$ is slightly larger than $\left|x_{2}\right|$ for $U / V g R=0.4$.

The exitation forces for incoming waves travelling against the current are displayed in figures $6(a, b)$. The vertical force is slightly larger than the horizontal force for $U \neq 0$. For $\mathrm{U} / \sqrt{\mathrm{gR}}=0.6$, the maximal force is about twice as large as the 


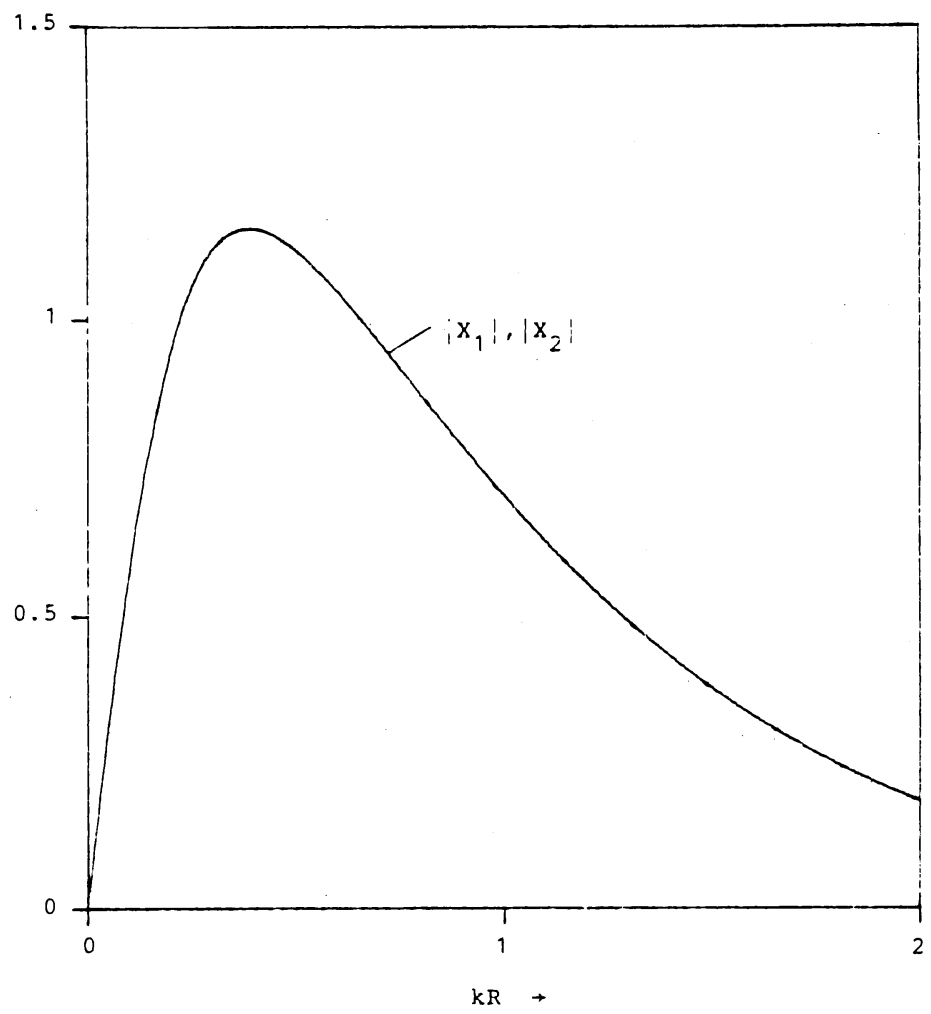

(a)

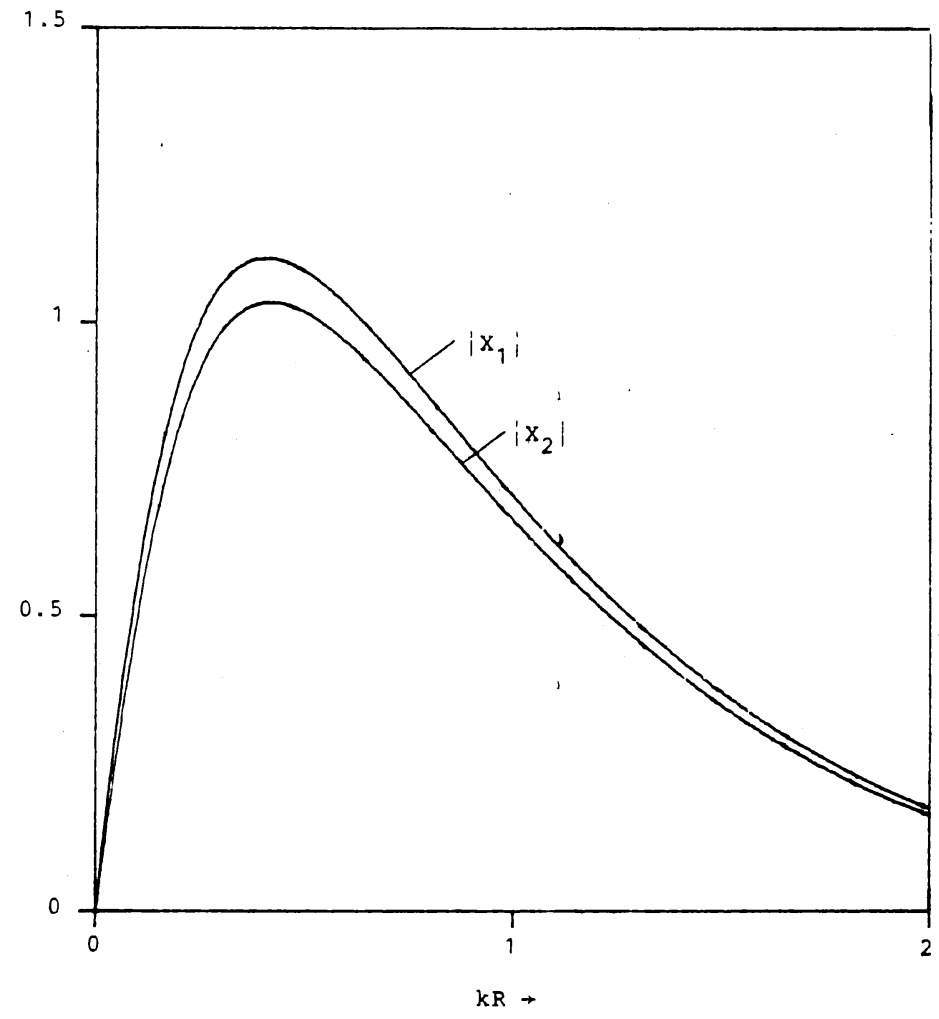

(b)

Figure 5. Horizontal and vertical exitation force for incoming waves travelling with the current, $d / R=2$, (a) $U=0$, (b) $U / \sqrt{g R}=0.4$. 
maximal force for $U=0$. For incoming waves with group velocity $\frac{1}{2} \sqrt{\mathrm{g} / \mathrm{k}}-\mathrm{U}$ approaching zero $(\tau \rightarrow \mathrm{l} / 4)$, the energy flux of the waves approaches zero. Hence, the periodic wave motion, the pressure at the cylinder, and the periodic pressure force vanish in this limit, as revealed in the figures $6(a, b)$.

The pressure distribution along the cylinder contour is displayed in figure 7 for incoming waves travelling with the current and in figure 8 for incoming waves travelling against the current. The pressure distributions are obtained when the maximal value of the exitation forces occur. For incoming waves travelling with the current, the maximal pressure is of the same magnitude for $U=0$ and $U / \sqrt{g R}=0.4$. For incoming waves travelling against the current, however, the maximal pressure for e.g. $U / \sqrt{g R}=0.6$ is four times the maximal pressure for $U=0$. The pressure distribution is symmetric about $\theta=0$ for $\mathrm{U} / \sqrt{\mathrm{gR}}=0,0.4$. For increasing $\mathrm{U}$, however, the distribution becomes asymmetric. Computations show that the first two terms in ( $3.3 a$ ) both give significant contribution to the exitation forces.

\section{Discussion.}

The forces in the radiation and diffraction problems are computed. The force due to the steady lee waves has been examined by Havelock (1936) and Tuck (1965). They found that this force is very small for small and moderate speed of the current. This means that for such values of $U$ the amplitude of the lee waves is vanishing. More precise, the lee waves may be neglected for $U / \sqrt{g R}<0.6$ when $d / R \geqslant 2$. 


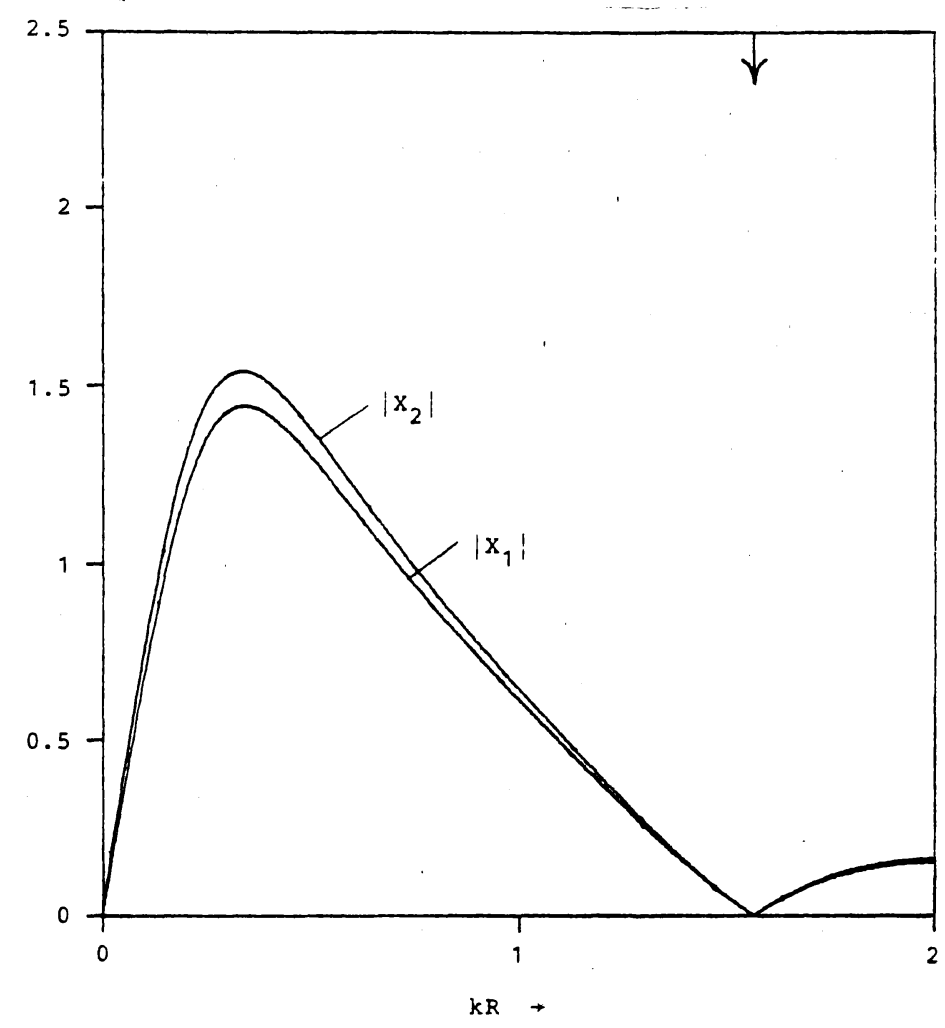

(a)

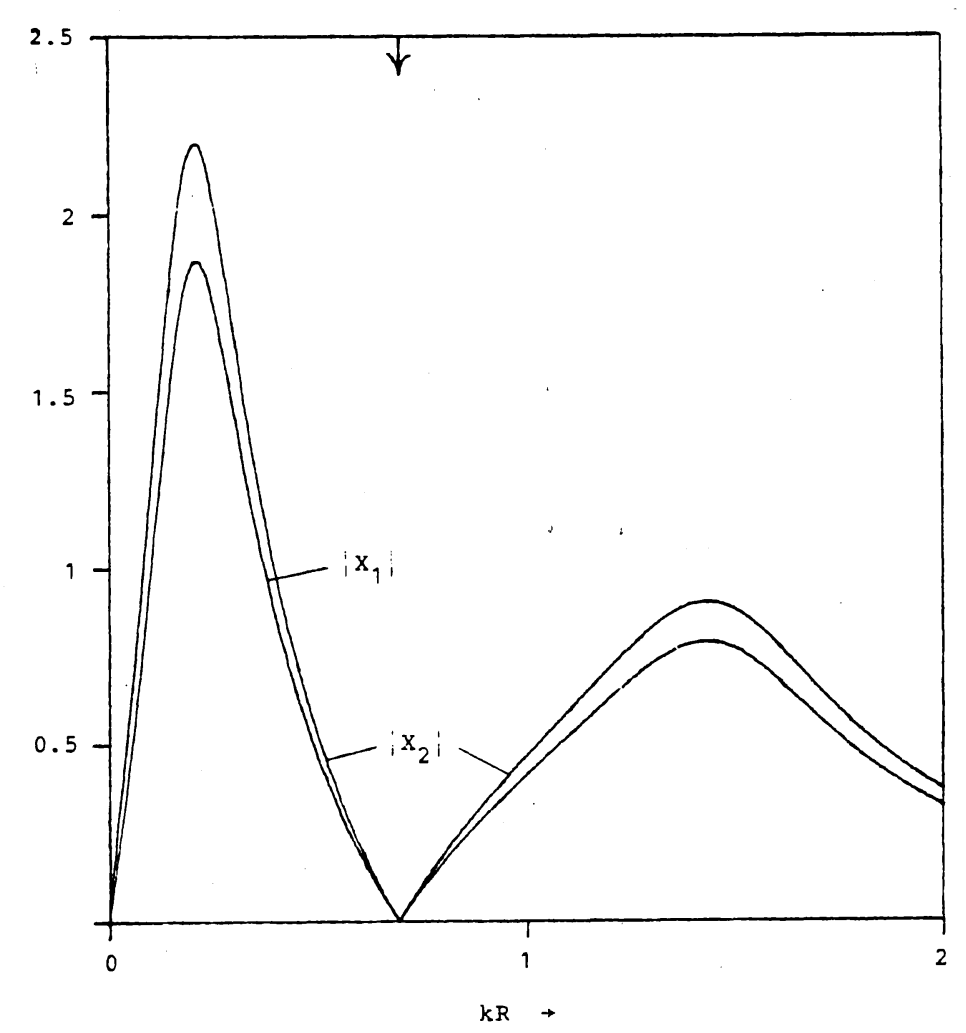

(b)

Figure 6. Horizontal and vertical exitation force for incoming waves travelling against the current, $d / R=2$, (a) $U / \sqrt{g R}=0.4$, (b) $U / \sqrt{g R}=0.6$. The small arrows denote the occurrence of zero group velocity of the incoming waves $(\tau=1 / 4)$. 


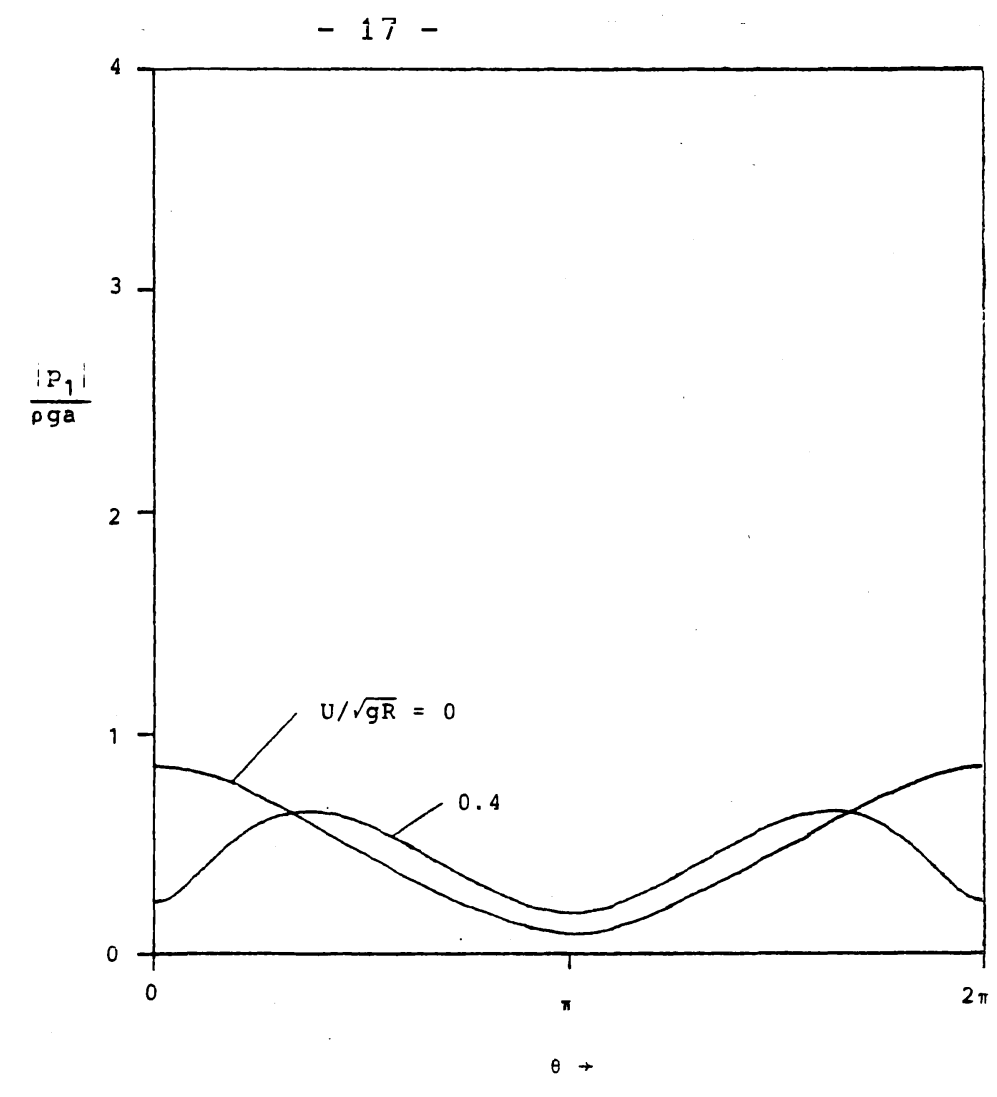

Figure 7. Pressure distribution along the restrained contour at the occurrence of maximal exitation force. The incoming waves are travelling with the current, $d / R=2, k R=0.4, U \sqrt{g R}=0,0.4$.

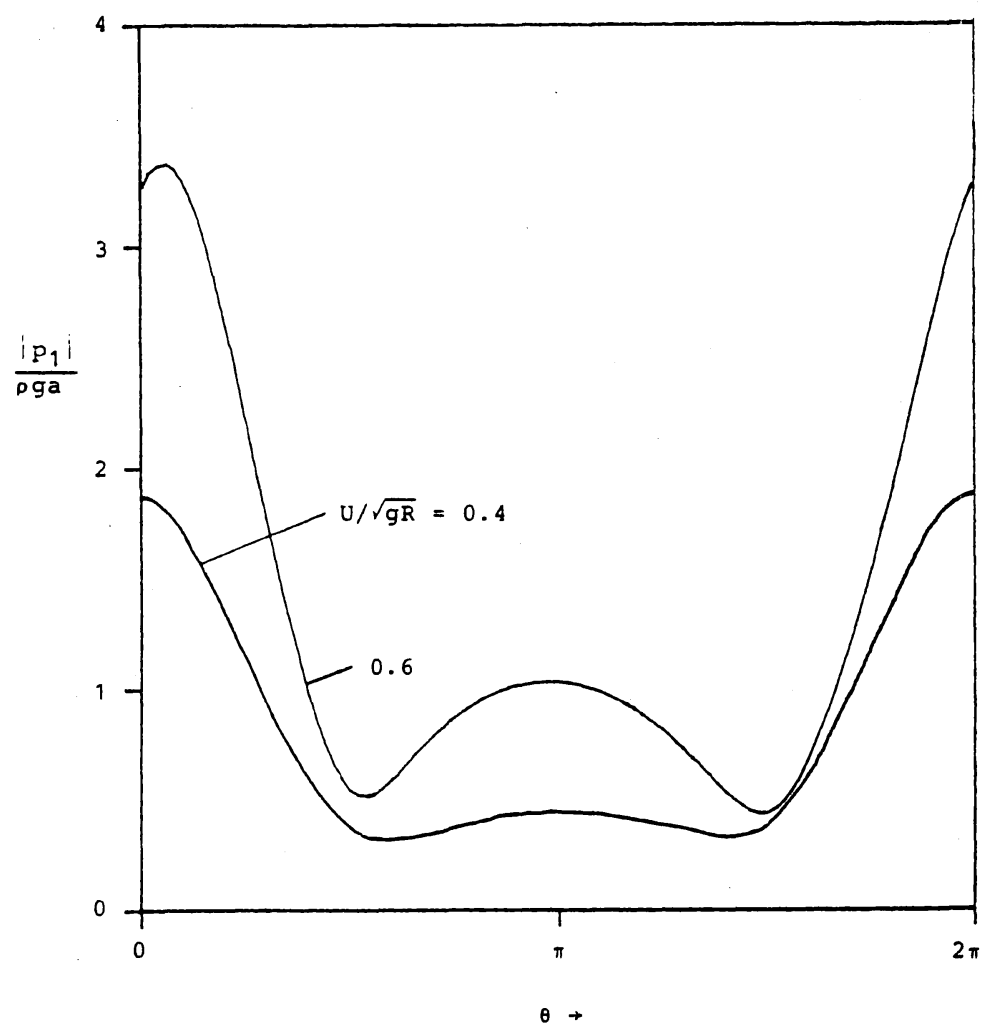

Figure 8. Pressure distribution along the restrained contour at the occurrence of maximal exitation force. The incoming waves are travelling against the current, $\mathrm{d} / \mathrm{R}=2, \mathrm{kR}=0.34$ and $\mathrm{U} / \sqrt{\mathrm{gR}}=0.4, \mathrm{kR}=0.21$ and $\mathrm{U} / \sqrt{\mathrm{gR}}=0.6$. 
However, even though the lee waves are absent, $\partial \chi / \partial x$ may not be small straight above the cylinder. Therefore, non-linear effects, which are not accounted for here, may possibly influence somewhat on the magnitude of the forces.

Effects due to vortex shedding are important. The horizontal drag force is given by

$$
D=\rho U^{2} R_{D}
$$

where $C_{D}$ is the drag coefficient. For large Reynolds number $C_{D}$ is of order unity. Comparing e.g. the maximal value of the exitation force $\hat{x}_{2}$ and $D$ we have for $U / \sqrt{g R}=0.6$ and $\mathrm{a} / \mathrm{R}=0.2$

$$
\hat{\mathrm{x}}_{2} / \mathrm{D}=1
$$

The lifting force due to the vortex shedding has a maximum amplitude which is of the same order of magnitude as the drag force. Hence, we conclude that the magnitude of the exitation force and the force due to vortex shedding are comparable for moderate speed of the current.

Acknowledgement.

The author wishes to thank Professor E. Palm for valuable discussions during the course of this work. 
References.

Dean, W.R. 1948. On the reflection of surface waves by a circular cylinder. Proc. Camb. Phil. Soc. 44, p. 483.

Grue, J. and Palm, E. 1984. Reflection of surface waves by submerged cylinders. Appl. Ocean Res. 6, no 1, p. 54.

Grue, J. and Palm, E. 1985. Wave radiation and wave diffraction from a submerged body in a uniform current. J. Fluid Mech. 151 , p. 257 .

Haskind, M.D. 1954. On wave motion of a heavy fluid. Prikl. Mat. Mekh. 18, p. 15.

Havelock, T.H. 1936. The forces on a circular cylinder submerged in a uniform stream. Proc. R. Soc. Lond. A 157, p. 526.

Lamb, H. 1932. Hydrodynamics, 6 th edn. Cambridge University Press.

Mo, A. and Palm, E. 1985. On radiated and scattered waves from a submerged elliptic cylinder in a uniform current.

(To appear).

Ogilvie, T.F. 1963. First- and second-order forces on a cylinder submerged under a free surface. J. Fluid Mech. 16, p. 451.

Sclavounos, P.D. 1984. The unified slender-body theory: Ship motions in waves. Proc. 15th Symp. Nav. Hydrodyn., Hamburg. Ursell, F. 1950. Surface waves on deep water in the presence of a submerged circular cylinder I and II. Proc. Camb. Phil. Soc. 46, p. 141 and p. 153.

Vada, T. 1985. A numerical solution of the second order, twodimensional wave diffraction problem for a submerged cylinder of arbitrary shape. Dr. Scient. thesis, Dept. of Mechanics, University of Oslo, Norway. 
